IdeAs

Idées d'Amériques

$7 \mid 2016$

Cinéma et histoire dans les Amériques

\title{
Le super-héros hollywoodien et l'ambiguité référentielle du désastre après le 11 septembre 2001
}

Hollywood Superheroes and the Referential Ambiguity of Disaster after 9/11

El superhéroe hollywoodense y la ambigüedad referencial del desastre después

del 11 de septiembre de 2001

O super-herói hollywoodiano e a ambiguidade referencial do desastre após o 11

de setembro de 2001

\section{Vincent Souladié}

\section{CpenEdition}

Journals

Electronic version

URL: https://journals.openedition.org/ideas/1548

DOI: 10.4000/ideas.1548

ISSN: 1950-5701

\section{Publisher}

Institut des Amériques

Electronic reference

Vincent Souladié, "Le super-héros hollywoodien et l'ambiguïté référentielle du désastre après le 11 septembre 2001", IdeAs [Online], 7 | 2016, Online since 01 July 2016, connection on 18 October 2022 URL: http://journals.openedition.org/ideas/1548 ; DOI: https://doi.org/10.4000/ideas.1548

This text was automatically generated on 18 October 2022

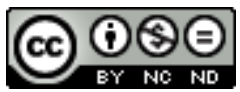

Creative Commons - Attribution-NonCommercial-NoDerivatives 4.0 International - CC BY-NC-ND 4.0 https://creativecommons.org/licenses/by-nc-nd/4.0/ 


\title{
Le super-héros hollywoodien et l'ambiguïté référentielle du désastre après le 11 septembre 2001
}

\author{
Hollywood Superheroes and the Referential Ambiguity of Disaster after 9/11 \\ El superhéroe hollywoodense y la ambigüedad referencial del desastre después \\ del 11 de septiembre de 2001 \\ O super-herói hollywoodiano e a ambiguidade referencial do desastre após o 11 \\ de setembro de 2001
}

Vincent Souladié

\section{Introduction}

1 Au lendemain du 11 septembre 2001, le milieu des comic books éprouva deux principaux types de réactions incarnées respectivement par DC Comics et Marvel : la mise en berne du récit super-héroïque, c'est-à-dire l'impuissance déclarée des éditeurs à « incorporer [à l'histoire] le tragique événement »' (Lance Eaton, 2009 : 109), ou bien la mise en crise de la figure super-héroïque, c'est-à-dire la participation fictionnelle du super-héros au deuil d'un événement qu'il n'a pas su empêcher, devenant une « figure fragile et sujette aux caprices de l'humanité $»^{2}$ (Lance Eaton, 2009: 109). Publié par DC Comics, 9-11: September 11th 2001 ne met exceptionnellement en scène aucun super-héros mais fait appel à une kyrielle d'auteurs maison pour relater les exploits de secouristes newyorkais, les véritables héros de la réalité. Publié par Marvel, Amazing Spider-Man vol. 2 voit son personnage principal se recueillir devant Ground Zero et prêter main forte aux sauveteurs. À Hollywood, en revanche, alors que la première vague de films de superhéros battait son plein, une logique de prudence encouragea provisoirement l'autocensure, et de nombreux films en cours de production se virent amputés de tout élément narratif ou visuel pouvant contrevenir à l'illusion de la fiction par leur rappel involontaire des événements du 11 septembre. Pour ce qui nous concerne, une scène 
des Indestructibles (The Incredibles, Brad Bird, 2004) fut coupée au montage : l'un des super-héros de l'intrigue y mesurait la portée de ses pouvoirs en provoquant par mégarde l'effondrement d'un immeuble abandonné. Pour Spiderman (Sam Raimi, 2002), c'est le matériel promotionnel qui fut corrigé. La première bande-annonce du film montrait l'homme-araignée piéger des criminels héliportés en tendant une toile entre les tours jumelles du World Trade Center.

2 L'analogie entre les images de l'attentat et l'ordinaire du spectaculaire hollywoodien semblait si évidente qu'elle imprégna nombre de regards spectatoriels. Stephen Prince cite des témoignages de critiques cinématographiques hantés par les images au point de repérer dans tous les films sortis après 2001 des réminiscences des attentats : « Le sentiment de saturation et de stupéfaction déclenché par le 11 septembre s'est révélé à la fois puissant et tenace, et les studios hollywoodiens ont naturellement pris peur à l'idée de se lancer sur ce terrain chargé d'émotions, où l'imagerie récente du 11 septembre était si intrinsèquement bouleversante $»^{3}$ (Prince, 2009 : 3). Le spectaculaire catastrophique retrouva pourtant vite ses droits, mais après la date fatidique du 11 septembre, tout cinéaste hollywoodien appelé à mettre en scène des séquences de destruction à grand spectacle prit le risque de se confronter à une comparaison, quasi inévitable désormais, avec le souvenir des attentats. Plutôt que le genre du cinémacatastrophe, c'est la fiction super-héroïque qui nous intéressera ici, pour l'engagement performatif de ses surhommes amenés à affronter des catastrophes à leur démesure. Nous souhaiterions montrer comment les situations spectaculaires dans lesquelles ces figures sont mises en scène les conduisent souvent à affronter des périls fictifs, spécifiques à la diégèse, mais dont la source référentielle semble indistinctement ancrée dans la réalité historique contemporaine. Le régime de représentation du spectaculaire commémore-t-il le souvenir des attentats de 2001 (résilience, revanche, catharsis) ou fait-il office d'agrément dramatique second pour définir subsidiairement l'identité du super-héros (coïncidence, stéréotype, amalgame)?

\section{Croisements référentiels}

3 La plupart des super-héros ont dépassé leur seule condition d'êtres de papier pour devenir des figures populaires de la culture anglo-saxonne avant même d'avoir vu leurs aventures transposées sur grand écran. Leurs caractéristiques identitaires n'échappent donc guère à la connaissance du public américain qui se déplace en salle pour admirer leurs exploits filmés. Comptant parmi les séquences récurrentes et attendues dévolues au genre, l'action hors-norme grâce à laquelle le personnage éponyme met en œuvre ses pouvoirs au service d'une action d'envergure, expose sa présence au monde diégétique et interagit avec lui à découvert et constitue en cela un épisode anticipé. Ses qualités physiques et morales, la nature de sa suprématie sur les hommes et les éléments, la connivence ou la défiance qu'il inspire, le concours qu'il apporte à la régulation de l'ordre, sont examinées par cette démonstration de force qui ancre sa figure superlative dans le monde diégétique.

4 Comme l'a par exemple relevé Stephen Keane, le film Superman (Richard Donner, 1978) fait intervenir le super-héros dans un contexte narratif et esthétique imprégné par les films-catastrophe, un genre très exploité dans les années soixante-dix, dont l'émergence fut souvent associée aux tourments sociologiques de l'époque, et qui à la fin de cette décennie se voit reformulé comme ici dans des blockbusters combinant 
action et science-fiction (Keane, $2006: 46$ ). Le déraillement d'un train et l'effondrement d'un barrage fourniront ainsi une double épreuve au héros en guise de climax final spectaculaire. Plus tôt dans le film, lorsque Superman sauve Loïs Lane de la chute d'un hélicoptère accidenté au sommet d'un gratte-ciel, la mise en scène énumère les tropes esthétiques récemment popularisés par La Tour Infernale (The Towering Inferno, John Guillermin, 1974) : l'énonciation anxiogène (contre-plongées spectaculaires de l'édifice rectiligne, miniaturisation de la victime en péril dans le point de fuite du cadre, plongées vertigineuses depuis le point de vue de cette dernière, reaction shots des badauds impuissants) et la stimulation nerveuse du découpage (chaque nouveau raccord semble annoncer la chute fatale de la victime mais chaque fois la retarde). Lorsque la figurine rouge et bleue de Superman apparaît dans le cadre, sa trajectoire dessine devant les arêtes verticales agressives de l'immeuble une sécante diagonale qui instaure un nouvel équilibre visuel. Par contraste, la mise en scène fait de lui le vecteur physique de l'optimisme en lui faisant interrompre le programme esthétique dépressif du film-catastrophe. Le super-héros intercepte sa promise dans sa chute, et lui apparaitt aussitôt comme un preux et séduisant bienfaiteur. Le cadrage centré de son buste dans le cadre en plan rapproché, le trait d'humour tranquille qu'il échange avec un passant avant de s'élancer, les plans de la foule en liesse, participent à façonner la figure d'un héros stable et rassurant, en accord avec les préceptes du comic book.

Depuis 2001, les "séquences de catastrophe-et-sauvetage " ${ }^{4}$ sont toujours employées pour illustrer l'action magistrale du super-héros, mais se voient parfois interprétées par certains commentateurs à la lumière de leur contexte socio-historiques et d'une possible référence aux événements tragiques du 11 septembre. Les grands édifices urbains mis en danger ainsi que la connivence du super-héros avec les pompiers, les militaires américains et la foule en liesse fournissent des figures narratives attendues qui constituaient déjà bien avant le 11 septembre l'ordinaire de la fiction superhéroïque, filmée ou dessinée. Pour autant, Sébastien Boatto envisage ces épisodes scénaristiques comme des « images-réparation [...] qui, inconsciemment, pansent les traumatismes occasionnés par le 11 septembre » (Boatto, 2009 : 130). Aussi enclin soit-il à généraliser «la forte signification politique" (Boatto, 2009: 130) de toutes ces séquences dans le cinéma de super-héros post-11 septembre, l'auteur montre pourtant que ce n'est pas tellement le contenu de l'action qui produirait cette référence, consciente ou non, mais surtout le régime esthétique qui lui est consacré. En l'occurrence, une séquence de sauvetage de Spiderman 3 (Sam Raimi, 2007), dans laquelle l'homme-araignée rattrape in extremis une jeune fille chutant du haut d'un gratte-ciel éventré, rappellerait par le choix de ses cadrages certains plans du documentaire 9/11: The Twin Towers (Richard Dale, 2006). Ajoutons que plusieurs plans en plongée sur les secouristes désarmés au pied de la tour semblent calquer la composition et la clarté lumineuse de quelques plans de World Trade Center (Oliver Stone, 2006). Le film affronterait donc moins les images du 11 septembre lui-même que ses refigurations fictionnelles. Or, par nécessité narrative, pour compléter les carences énonciatives des images en direct de l'événement, ces reconstitutions ont puisé leurs images dans un socle de poncifs eux-mêmes hérités du spectaculaire hollywoodien. Travaillé par le rappel de ces représentations, le souvenir de l'attentat se conjugue alors au récit super-héroïque comme une référence indirecte. Lorsque le corps plongeant de Spiderman, accompagné avec grâce par une caméra sur balancier, défie la pesanteur de la chute et s'immisce parmi les signes visuels de la catastrophe, l'articulation esthétique des deux régimes visuels nous semble fonctionner sur le même 
principe que pour le Superman de 1978: la puissance rassurante du super-héros est valorisée à l'écran par son contraste hyperbolique avec les éléments les plus pessimistes qui irriguent l'imaginaire contemporain (jadis le film-catastrophe, aujourd'hui les représentations du 11 septembre). C'est toujours à la croisée de ces régimes d'images partagées que le super-héros revendique la légitimité de sa présence à l'écran. Il construit son identité filmique en affrontant des périls à sa démesure, susceptibles de trouver un point d'accroche stimulant dans l'expérience spectatorielle.

\section{Insistance et mise à distance du souvenir}

Dans Superman Returns (Bryan Singer, 2006), le scénario transpose une péripétie du comics Man Of Steel, scénarisé et dessiné par John Byrne et Dick Giordano (1986). Lors de sa toute première apparition, le super-héros rattrape, retient, et dépose à bout de bras sur la pelouse d'un stade de baseball bondé une navette en détresse qui était prête à s'écraser sur les spectateurs. Kartik Nair affirme que cette séquence produit une " image audacieuse, arrivant à peine cinq ans après le 11 septembre " $^{5}$ (Nair, $2013: 21$ ), mais qu'elle se réapproprie le souvenir de l'attentat en garnissant l'image d'emblèmes nationaux (le sigle de la NASA, le stade de base-ball, les couleurs rouge et bleu du costume du sauveur, l'hymne national joué par la fanfare) et en ôtant «au 11septembre sa dimension urbaine $»^{6}$ (Nair, $\left.2013: 21\right)$. Dan Hassler-Forest avance pour sa part que cette « réécriture de la calamité $»^{7}$ déplace la menace aérienne dans un lieu de compétition sportive pour offrir à Superman un public diégétique prêt à applaudir l'exploit de son héros et encourager le spectateur à une participation émotionnelle (Hassler-Forest, 2012 : 60). De même, «le baseball incarnant un sentiment fortement transhistorique d'américanité', ce lieu entraîne des associations nostalgiques qui ramènent à un passé indéterminé défini comme celui de 'l'innocence', plutôt qu'aux notions de classes, de géopolitique, ou d'idéologie $»^{8}$ (Hassler-Forest, 2012: 60). Superman Returns convoquerait donc lui aussi le souvenir de l'attentat mais en le mettant tout de suite à distance par soustraction de réalité, dédramatisation et décontextualisation, et par hypertrophie des seules valeurs positives fédératrices qu'il a encouragées, la ferveur nationaliste et la célébration des héros, de façon à procurer un spectacle plaisant et impressionnant.

7 Toutefois, si les signes référentiels du 11 septembre sont occultés, comme le montrent ces deux auteurs, en quoi cette séquence se rapprocherait-elle encore du tragique événement plutôt que des morceaux de bravoure spectaculaires élaborés par Hollywood depuis des décennies? Il nous semble dans ce cas précis que le souvenir du 11 septembre, aussi difficile à éviter qu'il est difficile à mesurer pour les producteurs dès lors qu'est mobilisé ce genre de situation à grand spectacle, a pu servir de contrepoint à l'apparition super-héroïque pour offrir un climax d'envergure face auquel Superman gagne une puissance plus mesurée que triomphante. Dans cette séquence en effet, l'événementialité du corps hors-norme de Superman se dessine sous la forme évanescente d'un tracé pictural rouge et bleu traversant brièvement le cadre. Cette figuration plastique travaille le désir du spectateur à l'égard de la figure superhéroïque en retardant son entrée en scène, elle la révèle en même temps qu'elle la dissimule. Mais en comparaison avec le Superman de 1978, la mise en scène fait le choix de la modération, elle ne grave pas immédiatement à l'image l'icône magistrale, elle tarde à centrer son personnage dans le cadre, à lui définir une posture. Au corps totem 
de 1978 succède en 2006 un corps lacunaire et fragmentaire, d'abord une simple empreinte picturale et chromatique abstraite, une icône en devenir. La référence équivoque au 11 septembre ferait alors office d'agent dramatique propre à exposer le héros à un défi impérieux face auquel il ressort victorieux mais encore fragile, une caractéristique identitaire connue des lecteurs ${ }^{9}$, que le film prendra justement pour parti d'actualiser à l'écran à la faveur des signes les plus contemporains du danger.

\section{Spectacle et réalité du désastre}

Intrinsèquement lié à une ville qu'il a souvent pour charge de protéger, le super-héros y affronte tous les dangers la menaçant. La représentation de la catastrophe urbaine offre un pouvoir de sidération depuis toujours exploité par le cinéma. New York constitue en cela une cible de choix, régulièrement détruite par le cinéma-catastrophe (Deluge, Felix E. Feist, 1933), le cinéma de propagande anti-rouge (Invasion USA, Alfred E. Green, 1952), ou le cinéma de science-fiction (Independence Day, Roland Emmerich, 1996). Après le 11 septembre, elle sert de site d'accueil pour les combats à grande échelle des Quatre Fantastiques (Tim Story, 2004), de chacun des Spiderman (Sam Raimi, 2002, 2004 et 2007), de L'Incroyable Hulk (Louis Leterrier, 2008), de Watchmen (Zack Snyder, 2009), de Avengers (Joss Whedon, 2012), voire de Man of Steel (Zack Snyder, 2013), où la ville imaginaire de Metropolis décalque le modèle architectural de Manhattan. La déprédation urbaine souffre dans les premiers temps de la décennie d'une stratégie de l'esquive. Dans Les Quatre Fantastiques et Spiderman, le site d'accueil urbain reste encore intact, indifférent aux exploits physiques des héros éponymes. Plus tard, Les Quatre Fantastiques et le Surfeur d'Argent (Tim Story, 2007) et G.I. Joe (Stephen Sommers, 2010) délocalisent respectivement les scènes de catastrophe dans le centre de Londres et de Paris, et non pas sur le sol américain où se situe l'essentiel du récit. En 2009, Watchmen déréalise l'explosion nucléaire terroriste qui ravage Manhattan. Zack Snyder y choisit une iconographie-catastrophe elliptique, éloignée à la fois des images du 11 septembre et du dessin réaliste du roman graphique originel (Watchmen, Alan Moore et Dave Gibbons, 1987) : une lumière bleu pâle balaie les édifices qui se soulèvent au ralenti, figeant la représentation dans une violence uniquement suggérée.

Passage quasi obligé du récit super-héroïque hollywoodien, la séquence de catastrophe urbaine s'intensifie au fil des années et se voit progressivement gagnée par la ressemblance avec le modèle du 11 septembre. Symptomatiquement, la géographie de la ville est exploitée avec transparence dans Batman Begins (Christopher Nolan, 2005), Avengers et The Amazing Spiderman (Marc Webb, 2012), dans lesquels un immense gratteciel, chaque fois fictif, est la cible de forces maléfiques. Celui-ci est toujours épargné in extremis grâce à l'action des super-héros. Dans Wolverine (X-Men Origins: Wolverine, Gavin Hood, 2009), en revanche, l'affrontement final des deux clans rivaux de « mutants » se déroule sur le site de la centrale nucléaire américaine de Three Mile Island, dont les deux immenses cheminées s'effondrent verticalement dans un nuage de fumée grise, exactement cadré comme la chute médiatique des tours jumelles. Plus explicitement encore, dans Avengers et Man of Steel, la lutte intestine des surhommes en pleine ville multiplie les images de chaos urbain, explosion des façades d'immeubles sur lesquelles s'écrasent des projectiles volants, retournement des rues en nuages de gravats ou effondrement d'édifices publics. Plus de dix ans après les événements, le souvenir 
visuel du 11 septembre n'est plus évité, il configure plus explicitement l'imaginaire de la terreur dans lesquels s'inscrivent les fictions super-hérö̈ques.

Man of Steel imite les images tournées en direct le 11 septembre et les convertit en une somme de tics visuels : tremblé du cadre, zoom dans l'axe d'une explosion, imprécision de la mise au point, souillures de l'objectif par des particules de poussière grise, soit les effets d'une destruction montrée de façon oblique, une esthétique catastrophiste apparue avant le 11 septembre mais dont Geoff King a montré la montée en puissance dans le cinéma hollywoodien depuis 2001 (Geoff King, 2005 : 56). Le plus souvent saisi au vol par la caméra, le corps de Superman échoue à imposer dans le cadre sa stature iconique, il demeure le plus souvent en défaut d'incarnation. Alors qu'il lutte dans les airs contre sa nemesis, les gratte-ciels de la ville s'effondrent autour des civils, les piégeant sous les décombres et les laissant à leur propre sort, victimes d'un conflit auquel ils doivent résister par eux-mêmes. De la même façon, les derniers plans de Avengers montrent comment la population new-yorkaise se remet du cataclysme dont l'assemblée de super-héros vient de la sauver: des images télévisées diégétiques imitent les bulletins d'information post-11 septembre. On peut y voir les New-yorkais porter le deuil des victimes dans la rue puis remercier face à la caméra leurs mystérieux bienfaiteurs costumés. L'action spectaculaire fait coexister la dimension surhumaine des super-héros et leur impuissance à surmonter toute la réalité du drame. Leurs exploits positifs sont ainsi minimisés par l'ampleur du chaos funeste qu'il n'ont su prévenir. Si le super-héros a toujours pour fonction de rassurer le public, il gagne en consistance par humilité, sans priver les hommes de leur part tragique de réalité.

\section{Déchéance solennelle et résonance}

11 Dans The Dark Knight (Christopher Nolan, 2008), Batman se recueille en silence, impuissant et mélancolique, sur les ruines de l'édifice où sa promise vient de trouver la mort. Devant ce Ground Zero fictionnel, Christopher Nolan offre à la figure héroïque une plage de lyrisme neurasthénique. Là encore, ces ruines extériorisent la caractéristique identitaire principale de Batman, sa part tragique et pessimiste, en la croisant avec un régime visuel contemporain. Au sujet de Superman Returns, le cinéaste Bryan Singer confie avoir envisagé un moment de conduire le super-héros éponyme devant Ground Zero. "J'avais une scène dans le script que je n'ai pas tournée, et que je n'allais probablement jamais tourner, où Superman - après avoir volé dans la nuit pour secourir des gens - se serait dressé à l'aube devant Ground Zero. Il se serait tenu là comme pour dire "Si j'avais été présent, cela ne serait jamais arrivé" »10 (O'Sullivan, 2006). Le Superman de Singer ne se rend finalement pas à Manhattan dans le film, un lieu sans doute trop chargé historiquement pour justifier une réappropriation fictionnelle qui ne soit pas jugée déplacée. En revanche, le héros se trouve confronté à la menace d'un imposant îlot rocheux maléfique, flottant dangereusement dans l'océan Atlantique près des côtes américaines. À propos de la séquence où Superman se rend sur la surface de ce site sinistre, Dan Hassler-Forest remarque que « les images de ce continent désolé rappellent étrangement les photographies familières des décombres de Ground Zero $»^{11}$ (Hassler-Forest, $\left.2011: 144\right)$. Le héros vient affronter ici ses propres démons, et faillit devant ses adversaires. Bryan Singer cadre en plongée la minuscule silhouette du super-héros au centre de ce paysage excavé pour mieux nous donner à penser, à distance de son corps et de sa puissance figurative, tout son silence intérieur. 
Plus tard, en un geste final sacrificiel, le héros soulève et projette hors de la stratosphère cette masse nuisible, avant de laisser lourdement retomber sur Terre sous les yeux des humains sa silhouette fragile et déchue.

Selon la lecture métaphorique de Cynthia Erb, « le film envisage le site de Ground Zero comme une sorte de masse cancéreuse, et l'on souhaite que la masse soit amputée et jetée dans l'espace $»^{12}$ (Erb, 2009 : 245-246). Pourtant, plutôt que d'envisager ces films de super-héros comme des symptômes de l'actualité socio-historique, à laquelle ils viendraient apporter une réponse fictionnelle, il nous semble plus prudent de considérer que leurs analogies dramatiques et figuratives nourrissent l'imaginaire du film, lui donnent caution. Comme nous l'avions mentionné, les éléments dramatiques de Superman Returns et The Dark Knight cités ci-dessus sont partiellement transposés des comics, respectivement The Death of Superman et The Dark Knight Returns (Frank Miller et Klaus Janson, 1986). Les films ne constituent pas selon nous une métaphore de l'actualité, mais ils en absorbent, en exploitent, en redistribuent en revanche les schèmes figuratifs pour bâtir la fiction sur un espace iconographique partagé, dans lesquels les spectateurs peuvent reconnaître, de fait, l'humeur de leur temps. En somme, au lieu d'avancer que ces films auraient des choses à dire sur leur époque, il nous paraît plus déterminant de reconnaitre qu'ils offrent des outils d'expression figuratifs pour traduire aisément la fiction par l'actualité des images. Ainsi, la métaphore fonctionne dans le cadre diégétique, l'accablement du héros est identifié par l'avatar iconique de Ground Zero, espace familier dévasté qui l'accueille et l'oppresse, mais la réciprocité de cette métaphore, le film comme discours sur son époque, reste, à nos yeux, le résultat d'une instrumentalisation du discours filmique.

\section{Conclusion}

Il nous paraît en fin de compte plus probant de repérer combien la coïncidence de ces actions super-héroïques avec le 11 septembre ouvre l'image, de sorte que le stéréotype fictionnel est possiblement transformé par le discours critique en une réminiscence de l'événement. De même que les images médiatiques de la catastrophe ont un temps convaincu certains spectateurs qu'ils assistaient à une fiction, certains films d'après le 11 septembre semblent pouvoir convaincre symétriquement qu'ils rejouent la réalité. Plutôt que de considérer les images médiatiques des attentats du 11 septembre comme une matrice, il convient de se demander si elles n'agiraient pas plutôt comme un point de dérivation où les paradigmes de la réalité et de la fiction s'entrecroisent indistinctement. Après 2001, ce type d'action nous semble donc se soumettre à une ambiguïté référentielle. Tendue entre l'intention spectaculaire et l'intention discursive, entre la démesure ludique du divertissement et l'allégorie des angoisses culturelles contemporaines, l'action super-héroïque peut à la fois se référer au souvenir des attentats et faire lien, par nostalgie ou continuité, avec les traditions fictionnelles du passé. Hollywood semble chercher l'équilibre le plus adéquat pour que le souvenir du 11 septembre ne devienne pas inconvenant et pour que son oubli ne paraisse pas indécent. L'homologie, quand elle est reconnue, ne met pas forcément en danger l'investissement du spectateur dans la narration mais tend au contraire à convoquer la familiarité de son regard pour nourrir l'imaginaire fictionnel. Rechercher ce que cet univers filmique fictif pourrait avoir à nous communiquer sur notre réalité actuelle consiste selon nous en un retournement de problématique, puisqu'il ne s'agit pas 
vraiment ici de percevoir la réalité à travers la fiction mais de traduire la fiction avec les signes de la réalité que nous croyons percevoir dans l'image.

\section{BIBLIOGRAPHY}

\section{Ouvrages}

Boatto, Sébastien, « Du surhomme à Superman : évolution de la figure du héros dans le film d'action post-moderne ", in Claude Forest (ed.), Théorème, n 13, 2009, 121-131.

Eaton, Lance, « Spotlight Essay / Books : Comics Books », in Sarah E. Quayt et Amy M. Damico (dir.), September 11 in Popular Culture: A Guide, Santa Barbara : Greenwood, 2010.

Erb, Cynthia Marie, Tracking King Kong: A Hollywood Icon in World Culture, Detroit, Wayne State University Press, 2009.

Hassler-Forest, Dan, Capitalist Superheroes: Caped Crusaders in the Neoliberal Age, Alresford : Zero Books, 2012.

Hassler-Forest, Dan, « From Flying Man to Falling Man », in Véronique Bragard, Christophe Dony et Warren Rosenberg (eds.), Portraying 9/11. Essays on Representations in Comics, Literature, Film and Theatre, Jefferson, McFarland \& Company, Inc., 2011.

Keane, Stephen, Disaster Movies, The Cinema of Catastrophe, Londres : Wallflower Press, 2006 [2001] King, Geoff (dir.), The Spectacle of the Real, from Hollywood to Reality TV and Beyond, Bristol, Portland : Intellect, 2005.

Nair, Kartik, "Plummeting the pavement : the fall of the body in Spider-Man ", in Todd A. Comer et Lloyd Isaac Vayo (eds.), Terror and the Cinematic Sublime: Essays on Violence and the Unpresentable, Jefferson et Londres : McFarland \& Company, 2013, p. 15-28.

O'Sullivan, Michael, « For Singer, a Hero With Many Faces », The Washington Post, 30 juin 2006, consulté le 02 septembre 2012, http://www.washingtonpost.com/wp-dyn/content/article/ 2006/06/29/AR2006062900799.html

\section{Comic books}

9-11, September 11th 2001. The world's finest comic book writers and artists tell stories to remember (Collectif, DC Comics, 2002)

Amazing Spider-Man vol. 2 (John Romita Jr., Marvel Comics, 2001)

The Dark Knight Returns (Frank Miller et Klaus Janson, DC Comics, 1986)

The Death of Superman (Dan Jurgens, Roger Stern, Louise Simonson, Jerry Ordway, and Karl Kesel, DC Comics, 1992-1993)

Man Of Steel (John Byrne et Dick Giordano, DC Comics, 1986)

Watchmen (Dave Gibbons et Alan Moore, DC Comics, 1986-1987)

Filmographie 
11-Septembre - Dans les tours jumelles (9/11: The Twin Towers, Richard Dale, Dangerous Films, 2006)

The Amazing Spiderman (Marc Webb, Columbia Pictures, 2012)

Avengers (Joss Whedon, Walt Disney Studios Motion Pictures, 2012)

Batman Begins (Christopher Nolan, Warner Bros., 2004)

The Dark Knight (Christopher Nolan, Warner Bros., 2008)

The Dark Knight Rises (Christopher Nolan, Warner Bros., 2012)

Deluge (Felix E. Feist, RKO, 1933)

G.I. Joe : Le Réveil du Cobra ( G.I. Joe : The Rise of Cobra, Stephen Sommers, Paramount Pictures, 2010)

Indestructibles (The Incredibles, Brad Bird, Walt Disney Pictures, 2004)

L'Incroyable Hulk (The Incredible Hulk, Louis Leterrier, Marvel Studios, 2008)

Independence Day, (Roland Emmerich, 20th Century Fox, 1996)

Invasion USA (Alfred E. Green, Columbia Pictures, 1952)

Man of Steel (Zack Snyder, Warner Bros., 2013)

Les Quatre Fantastiques (Fantastic Four, Tim Story, 20th Century Fox, 2004)

Les Quatre Fantastiques et le Surfeur d'Argent (Fantastic Four : Rise of the Silver Surfer, Tim Story, 20th Century Fox, 2007)

Spiderman (Sam Raimi, Columbia Pictures, 2002)

Spiderman II (Sam Raimi, Columbia Pictures, 2004)

Spiderman III (Sam Raimi, Columbia Pictures, 2007)

Superman (Richard Donner, Warner Bros., 1978)

Superman Returns (Bryan Singer, Warner Bros., 2006)

La Tour Infernale (The Towering Inferno, 20th Century Fox \& Warner Bros., John Guillermin, 1974)

Watchmen (Zack Snyder, Warner Bros., 2009)

Wolverine (X-Men Origins: Wolverine, Gavin Hood, 20th Century Fox, 2009)

World Trade Center (Oliver Stone, Paramount Pictures, 2006)

\section{NOTES}

1. . « DC Comics acknowledged that they were not capable of incorporating the tragic event ». [Cette citation, ainsi que les suivantes, ont été traduites par l'auteur].

2. . "Marvel simply positions its superheroes as just as fragile and subject to the whims of humankind».

3. . " The feelings of being overwhelmed and stunned that $9 / 11$ triggered proved to be both powerful and tenacious, and the Hollywood studios were understandably fearful about intruding into such emotion-laden territory, one where the recent imagery of $9 / 11$ was so inherently upsetting ".

4. . « Disaster-and-rescue sequences ».

5. . « A bold image, coming merely five years after $9 / 11 »$.

6. . «Superman Returns has taken the urban out of $9 / 11 »$. 
7. . « Rewriting of calamity».

8. . « With baseball embodying a strongly transhistorical sense of "American-ness", the location activates associations of nostalgia that reach into a hazy past define in terms of "innocence" rather than class, geopolitics, or ideology ».

9. . The Death of Superman (Dan Jurgens, Roger Stern, Louise Simonson, Jerry Ordway, et Karl Kesel, 1992-1993).

10. . «I had a scene in the script which I never shot, and I probably was never going to shoot, where Superman would be standing - after flying around rescuing people at night - would be standing at dawn at Ground Zero. Sort of standing there, almost as if to say, 'If I had been here, this might not be.' ".

11. " "The images of this desolate continent seem eerily reminiscent of the familiar photos of the rubble at Ground Zero ».

12. . "The film envisions Ground Zero site as a cancerous mass and the wish is that the mass be cut out and hurled into space ».

\section{ABSTRACTS}

After 9/11, the figure of the superhero portrayed by Hollywood films was the subject of metaphorical readings, according to which the perilous situations depicted were seen to reflect contemporary cultural concerns and sometimes even to provide answers. Yet the representation of disaster has long been a transhistorical and transgeneric feature of both super-heroic fictions and Hollywood blockbusters. Reading too much meaning in these cinematic spectacles thus remains a highly questionable practice. As the figure of the superhero is typical of the ambiguous intersections between fiction and contemporary visual representations of reality, this article argues that the notion of referential ambiguity can serve to foreground the tension between oblivion and citation at stake in these Hollywood fictions, as well as the contradiction between establishing a distance with the event and exploiting the memory of it.

Après le 11 septembre 2001, la figure du super-héros mise en scène par Hollywood fait l'objet de lectures métaphoriques qui voudraient voir dans les périls catastrophiques qu'il affronte au cours de ses aventures des allégories ou des réponses aux angoisses culturelles contemporaines. La représentation $\mathrm{du}$ désastre constitue pourtant une constante transgénérique et transhistorique de la fiction super-héroïque comme du cinéma à grand spectacle américain. La surdétermination du spectaculaire contemporain par son contexte sociohistorique demeure ainsi relative et discutable. La figure du super-héros étant familière des croisements équivoques entre images de la fiction et images contemporaines de la réalité, la notion d'ambigüité référentielle voudrait ici mettre l'accent sur les tensions entre oubli et citation, mise à distance et instrumentalisation du souvenir de l'événement à l'œuvre dans ces fictions hollywoodiennes.

Después del 11 de septiembre de 2001, la figura del superhéroe puesta en escena por Hollywood ha sido objeto de lecturas metafóricas que querrían ver en los peligros catastróficos que el protagonista enfrenta a lo largo de sus aventuras, alegorías o respuestas a las angustias culturales contemporáneas. Sin embargo, la representación del desastre constituye una constante transgenérica y transhistórica tanto en las ficciones de superhéroes como en los grandes espectáculos cinematográficos estadounidenses. La sobredeterminación del espectáculo 
contemporáneo por su contexto socio-histórico resulta, por ello, relativa y discutible. En la figura del superhéroe son frecuentes los intercambios equívocos entre imágenes de ficción e imágenes de la realidad contemporánea, por ello, a través de la noción de ambigüedad referencial se buscará destacar las tensiones entre olvido y cita, distanciamiento e instrumentalización del recuerdo de los eventos, desarrolladas en esas ficciones hollywoodenses.

Depois do 11 de setembro de 2011, a figura do super-herói encenada por Hollywood tem sido objeto de leituras metafóricas que procurariam ver nos perigos catastróficos que ele enfrenta ao longo de suas aventuras, alegorias ou respostas às angústias culturais contemporâneas. Porém, as representações do desastre constituem uma constante trans-genérica e trans-histórica tanto das ficções de super-heróis como dos grandes espetáculos cinematográficos estado-unidenses. A sobredeterminação do espetáculo contemporâneo pelo seu contexto sócio-histórico é, por isso, relativa e discutível. Na figura do super-herói são frequentes os cruzamentos equívocos entre as imagens de ficção e as imagens da realidade contemporânea, dessa forma, através da noção de ambiguidade referencial se procurará dar destaque para as tensões entre esquecimento e citação, distanciamento e instrumentalização da lembrança dos eventos, veiculadas nas ficções hollywoodianas.

\section{INDEX}

Mots-clés: 11 septembre 2001, super-héros, Hollywood, catastrophe, spectaculaire, ville, ambiguïté référentielle

Palavras-chave: 11 de setembro de 2001, super-herói, Hollywood, catástrofe, espetacular, cidade, ambiguidade referencial

Palabras claves: 11 de septiembre de 2001, superhéroes, Hollywood, catástrofe, espectacular, ciudad, ambigüedad referencial

Keywords: September 11 2001, superheroes, Hollywood, disasters, spectacular, city, referential ambiguity

\section{AUTHOR}

\section{VINCENT SOULADIÉ}

Docteur en études cinématographiques, Vincent Souladié enseigne l'histoire et l'esthétique du cinéma à l'Université Toulouse II Jean Jaurès. Il y a soutenu une thèse portant sur l'esthétique du cinéma américain après le 11 septembre 2001. Ses recherches portent principalement sur l'identité formelle du cinéma américain contemporain, autour des questions de l'événement, de la catastrophe ou de la défiguration. Il a notamment consacré des articles à ces questions dans les revues Éclipses, Murmures et Entrelacs. 